\title{
Inseguridad alimentaria en los estados de México: un estudio de sus principales determinantes
}

\section{Food insecurity in Mexican states: a study on their major determinants}

\author{
Miguel Ángel Díaz-Carreño* \\ MAYTe SÁNChez-LeÓN** \\ Alejandra Díaz-Bustamente**
}

\begin{abstract}
This paper presents an analysis of the main explicative factors for the severe food insecurity in Mexican States, in order to do so we decided to estimate a multiple linear regression econometric model. The results show that the problem of severe food insecurity in the Mexican States in 2012 responds mainly to average schooling variables and the growth of the per capita primary sector production.
\end{abstract}

Keywords: severe food insecurity, Mexican states, average schooling, per capita primary sector production.

\section{Resumen}

Este artículo presenta un análisis de los principales factores determinantes de la inseguridad alimentaria severa en las entidades federativas de México, para lo cual se utilizó un modelo econométrico de regresión lineal múltiple. Los resultados sugieren que el fenómeno de la inseguridad alimentaria severa en los estados de México durante 2012 responde principalmente a las variables de escolaridad media y crecimiento de la producción per cápita del sector primario.

Palabras clave: inseguridad alimentaria severa, estados de México, escolaridad promedio, producción per cápita del sector primario.

* Universidad Autónoma del Estado de México, México. Correo-e: madiazc@uaemex.mx

** Egresada de la Universidad Autónoma del Estado de México, México. Correos-e: alejandra_bustamante2@hotmail.com; mayt_1102@hotmail.com 


\section{Introducción}

Las últimas cuatro décadas han significado para la población mexicana una profunda reducción de la capacidad adquisitiva de su ingreso, por arriba de $70 \%$, lo que en buena medida ha sido resultado de la elevada inflación anual registrada principalmente desde inicios de los ochenta, la cual en ocasiones se ubicó alrededor de 100\%: en 1982 fue de 98,8\%, en 1986 de 105,7\% y en 1987 de 159,2\% (Inegi, 2015). En este sentido, durante los años ochenta se agudizó el problema de la pobreza en México que persiste en la actualidad, por ejemplo, en 2010 y 2012 el porcentaje de la población en esta condición se ubicó en 46 y 45,4\% ${ }^{1}$, respectivamente, (Coneval, 2015a). El elevado crecimiento de los precios acompañado de constantes recesiones económicas y un lento crecimiento de los salarios contribuyeron en buena medida a agravar dicha problemática.

En este contexto, tras los efectos de la crisis económica de 1994-1995 el número de personas que se encontraba en situación de pobreza alimentaria era de 19 millones, cifra que se elevó hasta los 34,600,000 personas poco después de dicha crisis (Cabrera, 2009). No obstante, esta problemática ha evolucionado de forma muy heterogénea entre los distintos estados de México, al grado que para 2012 los estados que registraron mayores proporciones de inseguridad alimentaria severa fueron Tabasco y Campeche con porcentajes de $25,9 \%$ y $18,3 \%$, respectivamente, mientras que los estados con los menores niveles al respecto fueron Querétaro y Jalisco con 6\% y 7,5\%, respectivamente (Instituto Nacional de Salud Pública, 2012).

El fenómeno de la crisis alimentaria se agudizó en la década de los setenta, donde se experimentaron severos problemas de alimentación ocasionados principalmente por el alza de los precios internacionales en los granos. Es así que a nivel mundial y, en particular para países en desarrollo, se han realizado diversos estudios sobre la problemática de la inseguridad alimentaria con el fin de poder encontrar sus principales factores explicativos y, de esta forma, facilitar la generación de políticas públicas que contribuyan a su prevención. Al respecto, los trabajos de Sulo y Chelangat (2012), Hurtado (2011), Coordinación de Seguridad

\footnotetext{
${ }^{1}$ En situación de pobreza extrema, los porcentajes en 2010 y 2012 fueron de 10,4\% y 8,9\%, respectivamente (Coneval, 2015a). Una persona se encuentra en situación de pobreza multidimensional cuando no tiene garantizado el ejercicio de al menos uno de sus derechos para el desarrollo social (acceso a la educación, a los servicios de salud, a la seguridad social, calidad y espacios de la vivienda, servicios básicos en la vivienda y acceso a la alimentación), y si sus ingresos son insuficientes para adquirir los bienes y servicios que requiere para satisfacer sus necesidades. A su vez, la población en situación de pobreza multidimensional extrema, la cual dispone de un ingreso tan bajo que, aun si lo dedicase por completo a la adquisición de alimentos, no podría adquirir los nutrientes necesarios para tener una vida sana, además, presenta al menos tres de las seis carencias sociales (Coneval, 2015b).
} 
Alimentaria y Acceso a Oportunidades Económicas (2010), OSAN (2011), Zegarra y Tuesta, (2009), Ginja (2008) y Melgar et al. (2005), entre otros, han analizado el tema de la inseguridad alimentaria en varios países de América Latina y el Caribe tal es el caso de Colombia, Venezuela, Perú, Guatemala, entre otros.

De igual forma, para el caso de México diversos autores han analizado el tema de la seguridad alimentaria, destacan los estudios de Cuéllar (2011), Melgar et al. (2005) y Camberos (2000). Asimismo, sobresalen estudios de organismos nacionales e internacionales como el Consejo Nacional de Evaluación de la Política de Desarrollo Social (Coneval), la Secretaría de Salud (SSA), el Consejo Económico para América Latina (CEPAL), la agencia especializada de Naciones Unidas la Organización para la Alimentación y Agricultura (FAO), el Banco Mundial (BM), entre otros.

No obstante, gran parte de los estudios, tanto de carácter nacional como internacional, centran su atención únicamente en la oferta o disponibilidad de los alimentos, dando poca relevancia a la existencia de otros factores que sin duda contribuyen a determinar el nivel o grado de seguridad alimentaria en una población, tales como el poder adquisitivo de los consumidores a través de su ingreso o sus capacidades de elección para el mejoramiento de su bienestar alimenticio, entre otros.

En este sentido, el objetivo de esta investigación es analizar y medir el efecto que en términos medios tienen variables como la inflación en alimentos, el grado de escolaridad, el salario real, la tasa de desocupación respecto a la población económicamente activa (PEA), la tasa de ocupación en el sector informal, el crecimiento del producto interno bruto (PIB), los ingresos de la población a partir de los primeros deciles y el comportamiento de la producción del sector primario per cápita en la inseguridad alimentaria severa en los estados de México mediante la elaboración de un modelo econométrico.

Este artículo se divide en tres apartados; primeramente se aborda un marco conceptual para definir y argumentar sobre los principales factores que ha establecido la teoría como causales del fenómeno de la inseguridad alimentaria. Posteriormente, se describe la metodología empleada para la modelación estadística de la inseguridad alimentaria severa en los estados de México y, finalmente, se discuten los resultados obtenidos a partir del modelo econométrico estimado para posteriormente establecer las conclusiones de la investigación. 


\section{Marco conceptual y revisión de literatura}

Existen numerosas definiciones sobre inseguridad alimentaria debido a la complejidad y amplia cantidad de factores que explican a la misma. Por ejemplo, la FAO considera que la seguridad alimentaria existe cuando todas las personas tienen, en todo momento, acceso físico, social y económico a alimentos suficientes, inocuos y nutritivos que satisfacen las necesidades energéticas diarias y preferencias alimentarias para llevar una vida activa y sana (FAO, 2011). A su vez, el organismo establece que existe inseguridad alimentaria cuando las personas no tienen acceso físico, social o económico suficiente a los alimentos.

Por otra parte, la inseguridad alimentaria de acuerdo a la Escala Latinoamericana y Caribeña de Seguridad Alimentaria (ELCSA) se clasifica en los siguientes niveles: inseguridad alimentaria leve que se presenta cuando se ha experimentado una disminución en la calidad de la alimentación; inseguridad alimentaria moderada cuando hay una disminución de la cantidad de alimentos y, finalmente, la inseguridad alimentaria severa cuando un adulto o nińo no ingiere alimento en todo un día.

En esencia se pueden observar dos posturas en torno al tema de la inseguridad alimentaria. La primera se centra en la disponibilidad de los alimentos, es decir, la oferta; se toma a esta variable como la más importante para explicar la inseguridad alimentaria. La segunda postura hace referencia al ingreso como la variable sustancial en la explicación de la inseguridad alimentaria.

Respecto al primer planteamiento Malthus escribió en su primer ensayo sobre el principio de la población (1798), que la población crecía geométricamente y la producción de alimentos aritméticamente, es decir, que la población crecía más rápido, por consecuencia, la humanidad estaría condenada a sufrir sobrepoblación y escasez. La solución que propuso consistió en adoptar medidas de control de la población. Al respecto, Nikos y Bruinsma (2012) han establecido que a nivel mundial la producción agrícola puede incrementarse lo suficiente para satisfacer la demanda adicional proyectada a 2050; así, se espera que el producto agrícola global se incremente $60 \%$ por arriba de los niveles de $2005 / 2007$, teniendo en cuenta tanto la producción de alimentos como la no alimentaria.

Sin embargo, puesto que la disponibilidad de recursos, del ingreso y el crecimiento poblacional presentan una marcada distribución desigual, la seguridad alimentaria continuaría representando un serio desafío en el mundo. De esta manera, algunos países tendrían que aumentar su demanda efectiva de alimentos más rápidamente que en el pasado, fundamentalmente a partir de un crecimiento económico sostenible. Tales 
países son aquellos que se caracterizan por registrar altos niveles de pobreza y un elevado crecimiento de su población.

En este sentido, Camberos (2000) evalúa el grado de seguridad alimentaria que ha tenido México de 1950 a 1990 centrando su estudio en cambios de la estructura de la población, cambios en la demanda de alimentos, la producción y disponibilidad de los mismos. Dicho autor encuentra que sólo en 1980 se consigue la seguridad alimentaria y que el nivel de importación de granos fue un factor determinante para garantizar dicha seguridad alimentaria en ese año, al presentarse un volumen de importación de $29 \%$ del total de la oferta de granos.

Por su parte, Torres (2002) trató de medir la dimensión de la seguridad alimentaria con base en mapas regionales de alimentación a una escala subestatal e incluso municipal para los 32 estados de la república. La primera condición que tomó para definir un mapa de seguridad alimentaria fue la producción de alimentos, siendo el PIB de la agricultura dividido entre el número de habitantes de cada estado el factor que explica mejor la seguridad alimentaria de las entidades en términos de su oferta, ya que muestra la producción promedio de cada habitante.

Sin embargo, el autor también menciona que aquellas regiones que cuentan con potencial agropecuario importante no necesariamente se encuentran en una situación de consumo alimenticio privilegiado. De igual forma, argumenta que la situación de vulnerabilidad alimentaria de las regiones de México tiende a ubicarse más en las posibilidades de acceso real, toda vez que el nivel de ingreso de la población lo condiciona y éste presenta un grave deterioro acumulado que impide aumentar y diversificar el consumo alimentario.

En dicha investigación, las entidades en las que más de $80 \%$ de su población tiene ingresos menores a tres salarios mínimos y se encuentran en la situación más crítica de seguridad alimentaria son Oaxaca y Chiapas. Torres (2002) llega a tal conclusión tomando en cuenta el valor de la llamada "canasta normativa alimentaria" $\left(\mathrm{CNA}^{2}\right)$ que para 1990 fue equivalente a 3,2 salarios mínimos.

De esta forma, Torres (2002) establece que la oferta alimentaria desempeña un papel importante, ya que mediante esta variable identifica geográficamente a las regiones con suficiente o insuficiente producción agrícola, aunque este factor no sea el único determinante en el grado de seguridad alimentaria que se presenta en las regiones. Es así que la relevancia de este estudio radica en la inclusión de la variable ingreso en la explicación de la problemática analizada.

\footnotetext{
${ }^{2}$ Integrada por 34 productos alimenticios básicos con las cantidades y combinaciones adecuadas, que satisfacen el requerimiento mínimo diario (sugerido por el Instituto de Nutrición) de 2082 calorías y 35,1 gramos de proteínas por adulto (Cabrera et al., 2005).
} 
Por otra parte, la Encuesta Nacional de Salud y Nutrición (Ensanut), que aborda tanto el estudio nutricional de la población como la situación de salud desde 2006, mide el estado de inseguridad alimentaria usando la versión adaptada para México de la ELCSA, la cual consta de 15 preguntas dirigidas al jefe de familia o a la persona encargada de preparar los alimentos en el hogar. Asimismo, la escala clasifica a los hogares en cuatro categorías dependiendo del número de respuestas positivas y si cuentan, o no, con integrantes menores de 18 años (Secretaría de Salud, 2012).

Los hogares que se clasifican en la categoría de inseguridad alimentaria leve informan, en primera instancia, preocupación por el acceso a los alimentos, sacrificando la calidad de la dieta. Cuando los hogares se encuentran en inseguridad alimentaria moderada, además del sacrificio en calidad, refieren restricciones en la cantidad de alimentos consumidos. Los hogares en inseguridad alimentaria severa, además de las percepciones anteriores, relatan experiencias de hambre en adultos y finalmente en niños.

La importancia de la Ensanut 2012 en la evaluación de la inseguridad alimentaria en el país radica en la evidencia arrojada por sus resultados. Esta indica que una proporción de 30\% de hogares se ubicó en seguridad alimentaria; mientras que $70 \%$ se clasificaron en alguna de las tres categorías de inseguridad alimentaria: $41,6 \%$ en inseguridad leve, $17,7 \%$ en inseguridad moderada y, $10,5 \%$ en inseguridad severa.

La aportación de la Ensanut 2012 en el estudio y medición de la inseguridad alimentaria en México se da en la escala de preguntas utilizada, la cual además de categorizar a dicha problemática, también deja entrever las posibles causas por las que una persona calificada dentro de inseguridad alimentaria moderada y severa, disminuye la cantidad en el consumo de alimentos o, en el peor de los casos, deje de ingerir alimentos un día entero.

Entre los múltiples estudios que se han realizado para el análisis de la seguridad alimentaria en México, se encuentra también el informe elaborado en 2010 por el Coneval, dentro del cual se da paso a un elemento adicional, la estabilidad temporal, ya que la permanencia de la problemática es un factor adicional de la seguridad alimentaria en México.

Por lo anterior, el organismo elabora una clasificación de inseguridad alimentaria que permite conocer la temporalidad del fenómeno en los hogares mexicanos (Coneval, 2010).

Las personas en inseguridad alimentaria crónica son aquellas cuyo consumo alimentario está por debajo de los requerimientos necesarios o que padecen hambre de forma persistente durante periodos largos, ocasionados por reducciones estacionales en la producción o abasto de alimentos, o del empleo. 
En cambio, aquellos en inseguridad alimentaria estacional y transitoria son los que se enfrentan a niveles de consumo inferiores a los adecuados por periodos definidos, como resultado de choques económicos y naturales, variaciones en los precios internacionales, sequías y cualquier tipo de desastre natural, e incluso por periodos de enfermedad o desempleo temporal que ocasione disminución en su ingreso.

Esto indica que un hogar se considera en seguridad alimentaria si tiene protección contra ambos tipos de inseguridad. Como se observa, el estudio puntualiza la importancia de dos de las variables más relevantes que definen el índice de inseguridad alimentaria: oferta e ingreso.

De igual manera, se establece que para medir la situación de riesgo de seguridad alimentaria de un país, además de los indicadores de disponibilidad de alimentos y de desnutrición, también se deben incorporar indicadores de crecimiento económico, pobreza, salud, entre otros (Coneval, ${ }^{3}$ 2010). Adicionalmente, dentro de los indicadores para la dimensión de acceso o capacidad para adquirir alimentos, el organismo refiere que el acceso económico se relaciona con el precio de un alimento en particular o el precio relativo de alimentos alternativos o sustitutos, así como con las restricciones presupuestarias de los consumidores. ${ }^{4}$

Por su parte, Torres (2003) establece que existe una amplia gama de factores explicativos de la inseguridad alimentaria, los cuales identifica como coyunturales y estructurales: En el primer caso ubica a los i) fenómenos climáticos adversos, ii) fluctuaciones en los ingresos en divisas o en la capacidad para importar, iii) malas cosechas no compensadas por mejores precios, iv) caídas estacionales de los precios del producto, $v$ ) desempleo estacional, vi) declinación temporal en los salarios reales y, vii) la transición hacia otras opciones productivas. En tanto que entre los factores estructurales se encuentra el $i$ ) deterioro del potencial productivo (salinización, erosión, desertificación, etc.), ii) sustitución de cultivos alimentarios, iii) deterioro de los términos de intercambio, iv) falta o insuficiencia de tierra laboral, v) salarios menores al costo de la canasta

\footnotetext{
${ }^{3}$ Para medir las dimensiones de seguridad alimentaria en México el Coneval, en coordinación con otras instituciones, realiza la elaboración y aplicación de encuestas como: Encuesta Nacional de Ingresos y Gastos de los Hogares (ENIGH), a través del Inegi: Módulo de Condiciones Socioeconómicas de la ENIGH; Encuesta Nacional de Salud y Nutrición (Ensanut), por el Instituto Nacional de Salud Pública y la Secretaría de Salud; la Encuesta Nacional de Abasto Alimentación y Estado Nutricional en el Medio Rural (ENAAEN), por Coneval; por mencionar algunas.

${ }^{4}$ Los resultados arrojados por el análisis de las encuestas ENIGH, Ensanut y ENAAEN muestran que el acceso y capacidad de adquirir los alimentos dependen de un gran número de factores que tienen que ver con los mercados de alimentos y con la capacidad de pago de los consumidores (Coneval, 2010). En 2008 se estimó que alrededor de 3,800,000 hogares no cuentan con el ingreso suficiente para comprar los bienes de la canasta básica, toda vez que en los últimos ańos el precio de la canasta ha aumentado de forma considerable y con ello la pérdida del poder adquisitivo, lo que, acorde con los análisis del Coneval (2010), ha tenido un gran impacto en la evolución de la inseguridad alimentaria del país.
} 
básica, vi) desempleo crónico sin seguro social compensatorio, vii) aislamiento geográfico, viii) analfabetismo, ix) problemas de salud y edad, entre otros.

Es claro que Torres (2003) destaca como factores explicativos del fenómeno de la inseguridad alimentaria, principalmente, a las diversas problemáticas que enfrenta la producción del sector agropecuario, sobre todo en el mundo en desarrollo tanto de tipo coyuntural como estructural, a las fluctuaciones del tipo de cambio de los términos de intercambio y a los salarios reales.

Los diversos estudios tanto de carácter teórico como aplicado sugieren que el tema de la inseguridad alimentaria debe ser abordado y explicado a la luz de una gran cantidad de factores determinantes: insuficiente oferta de alimentos que no logra cubrir una demanda creciente, crecimiento económico y del empleo formal insuficientes, niveles de ingreso reducidos, aumentos elevados en los precios de los alimentos, pobreza y salud, entre otros.

En esta investigación se destaca el carácter multifactorial del fenómeno de la inseguridad alimentaria, por lo que además de reconocer que dicha problemática está asociada a los indicadores económicos antes referidos se incorpora a la educación como una variable explicativa fundamental de la inseguridad alimentaria de acuerdo al enfoque de capabilities o "capabilidades" 5 expuesto por Sen $(1981,1983)$. Dicho enfoque considera que las personas tienen el potencial o "capabilidad" para existir o actuar, o bien, que los seres humanos poseemos un gran número de potencialidades que las situaciones adversas nos impiden desarrollar; por lo que cualquier principio de distribución debe centrarse en la mejora de las circunstancias que permiten desarrollar las "capabilidades" (Nebel et al., 2014).

El enfoque teórico de capabilities establece que el bienestar de las personas no depende fundamentalmente de sus niveles de ingreso sino del grado de desarrollo de sus "capabilidades". Nussbaum (2000, 2003) propuso una lista concreta de capabilities la cual se compone de las siguientes 10 categorías: $i$ ) vida (no morir de forma prematura, vivir una vida de duración normal), ii) salud física (mantener una buena salud en general y recibir una alimentación adecuada), iii) integridad física (vivir sin peligros), iv) sentido, imaginación y pensamiento (tener acceso a la educación y formación científica), $v$ ) emociones (sentir emociones por cosas y personas externas o por uno mismo), vi) razón práctica (reflexionar acerca de la planificación de la propia vida), vii) afiliación (poder

${ }^{5}$ La "capabilidad" corresponde al conjunto de funcionamientos entre los cuales el individuo podrá elegir los que quiere realizar para alcanzar la vida que desea o mejorar su bienestar. Si los funcionamientos son los que el individuo realiza, la "capabilidad" representa la libertad efectiva que posee el individuo para realizar los funcionamientos que él estima bueno realizar (Nebel et al., 2014). 
vivir por y para los demás), viii) otras especies (vivir con el resto de los seres vivos en un entorno natural y de respeto), ix) juego (dedicar tiempo a actividades recreativas), $x$ ) control del entorno (participar de forma efectiva en las decisiones políticas que afectan directamente nuestra vida (Robeyns, 2005; Alarcón y Guirao, 2013). En este sentido, Alarcón y Guirao (2013) destacan que en su gran mayoría estas capabilities sólo pueden ser alcanzadas a través de la educación. De esta manera, en este estudio se considera a la educación o "escolaridad promedio" de los individuos como una variable proxy de las capabilities referidas con anterioridad.

\section{Metodología}

Este apartado tiene la finalidad de describir la metodología estadística y econométrica que facilitó la elaboración del modelo de la inseguridad alimentaria severa (IAS) en los estados de México. Se comentan también los conceptos empleados en el análisis de los modelos de regresión lineal (MRL) y del método de mínimos cuadrados ordinarios (MCO).

El MRL empleado en esta investigación se puede representar a partir de la siguiente ecuación.

$$
Y=\beta_{0}-\beta_{1} X_{1}-\beta_{2} X_{2}-\beta_{3} X_{3}+\cdots+\beta_{11} X_{11}+\varepsilon
$$

Donde $\mathrm{Y}$ es el vector de observaciones relativas al porcentaje de los hogares en condición de IAS en los distintos estados de México durante 2012 , en tanto que $\mathrm{X}_{1}, \mathrm{X}_{2}, \ldots, \mathrm{X}_{11}$ son vectores referentes a las variables explicativas del modelo que son descritas más adelante; el término $\varepsilon$ se refiere a la perturbación estocástica del modelo.

La inflación en alimentos $\left(\mathrm{X}_{1}\right)$ es la tasa de crecimiento del índice nacional de precios al consumidor (INPC) de los alimentos, comprende el periodo 2000-2012 para cada uno de los estados de México y es publicado por el Inegi (2013a).

La escolaridad $\left(\mathrm{X}_{2}\right)$ es el número promedio de años de escolaridad durante 2012 de cada estado de la república mexicana y la información fue obtenida del Inegi (2012).

El salario real $\left(\mathrm{X}_{3}\right)$ es el salario mínimo nominal que publica la Comisión Nacional de Salarios Mínimos (Conasami, 2013) dividido por el INPC general, tomando en cuenta el periodo 2000-2012 para cada uno de los estados del país mexicano.

${ }^{6}$ Las diferentes variables empleadas para la modelación econométrica de esta investigación tienen periodos diferentes con base en la disponibilidad de la información correspondiente. 
La tasa de desocupación $\left(\mathrm{X}_{4}\right)$ es el porcentaje de desocupación respecto a la población económicamente activa (PEA) durante el periodo 20002012 para cada uno de los 32 estados mexicanos. Las tasas de desocupación utilizadas en este estudio fueron obtenidas del Inegi (2013b).

La tasa de ocupación en el sector informal $\left(\mathrm{X}_{5}\right)$ es el porcentaje de ocupación en el sector informal respecto a la PEA en 2012 para cada uno de los estados de México y fue publicada por el Inegi (2013b).

El crecimiento del PIB estatal $\left(\mathrm{X}_{6}\right)$ es la tasa de crecimiento del producto interno bruto (PIB) durante el periodo 2000-2011 para cada uno de los estados mexicanos y representa el comportamiento de la actividad económica estatal en dicho periodo (Inegi, 2013c).

La producción del sector primario per cápita $\left(\mathrm{X}_{7}\right)$ es la tasa de crecimiento del PIB del sector primario per cápita durante 2005-2010 de cada uno de los estados. Dicho indicador representa el crecimiento de las actividades económicas primarias por habitante en el periodo establecido (Inegi, 2013c).

Los deciles de ingreso de la población I, II, III y IV $\left(\mathrm{X}_{8}, \mathrm{X}_{9}, \mathrm{X}_{10} \mathrm{y}_{11}\right)$ representan las tasas de crecimiento durante 2005-2010 para dichas variables de cada uno de los estados. Estos indicadores muestran los niveles de ingreso más bajos de la población. De hecho los deciles I, II y III corresponden a un nivel de ingreso menor que un salario mínimo; en tanto que los deciles IV, V y VI representan un ingreso de más de un salario mínimo pero menor a dos salarios mínimos (Inegi, 2013d).

La información acerca de las variables descritas se resume en el cuadro 1.

El cuadro 1 muestra que los estados con mayor grado de IAS en México son Tabasco, Campeche, Guerrero, Michoacán y Chiapas; mientras que los estados que presentan un grado menor de inseguridad alimentaria son Querétaro, Jalisco, Yucatán y Nuevo León. Un primer análisis con base en la información del cuadro 1 permite observar que aquellos estados con mayores niveles de inseguridad alimentaria severa no son precisamente los que han mostrado mayores niveles inflacionarios en el rubro de alimentos en el pasado reciente. Este es el caso de Tamaulipas, Colima y Nayarit, quienes presentaron los mayores aumentos del índice referido durante el periodo 2000-2012, y que sin embargo, sus niveles de IAS se ubican por debajo de la media nacional.

En este mismo sentido llama la atención el caso de los estados de Tabasco, Campeche y Guerrero que por un lado presentan los porcentajes de IAS más elevados, no obstante, se acompañan de niveles inflacionarios en alimentos cercanos a los de la media nacional. Lo que de antemano sugeriría una débil relación entre ambas variables, o bien, que los fuertes incrementos en los precios de alimentos no sería uno de los principales 


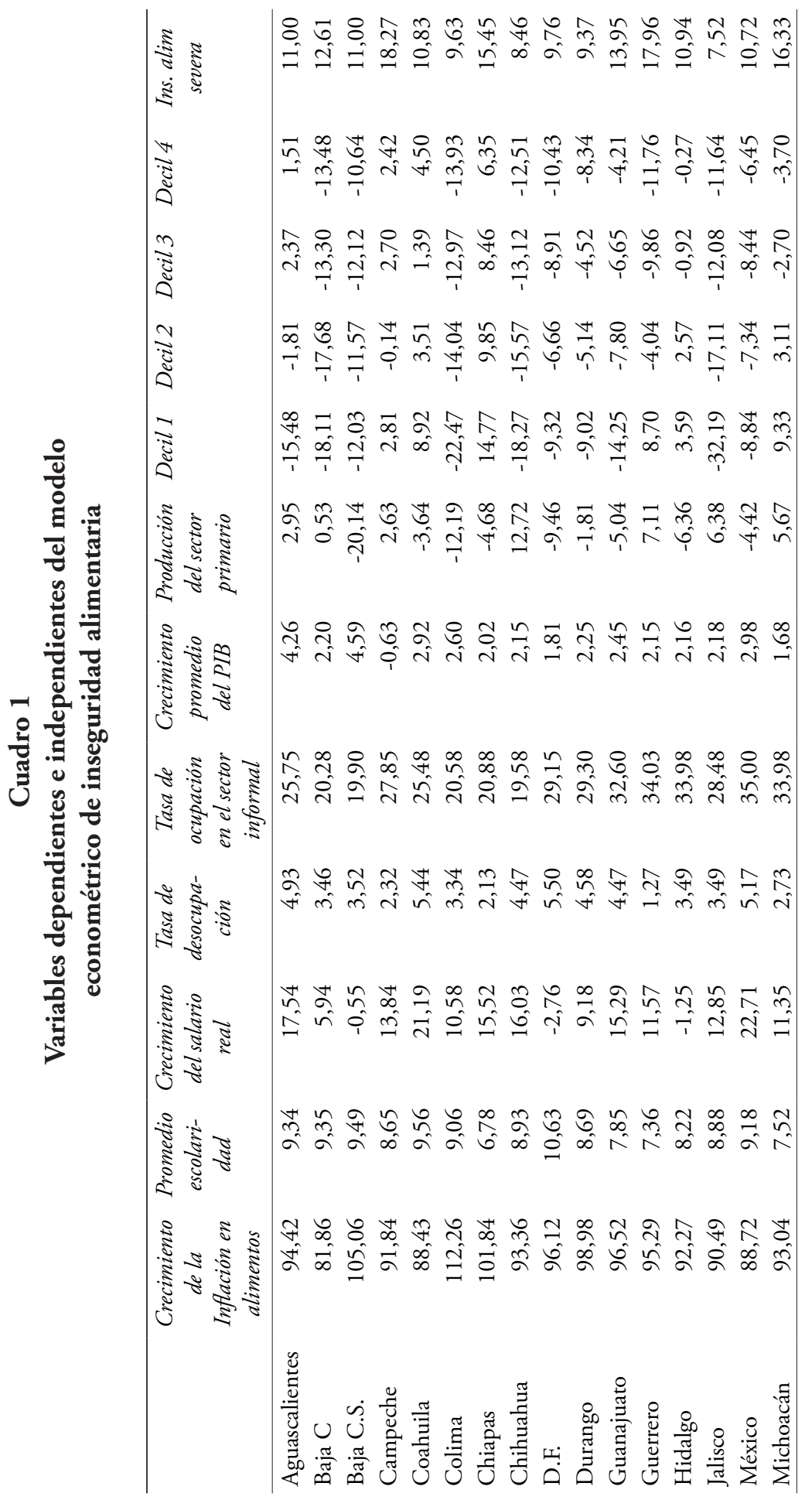




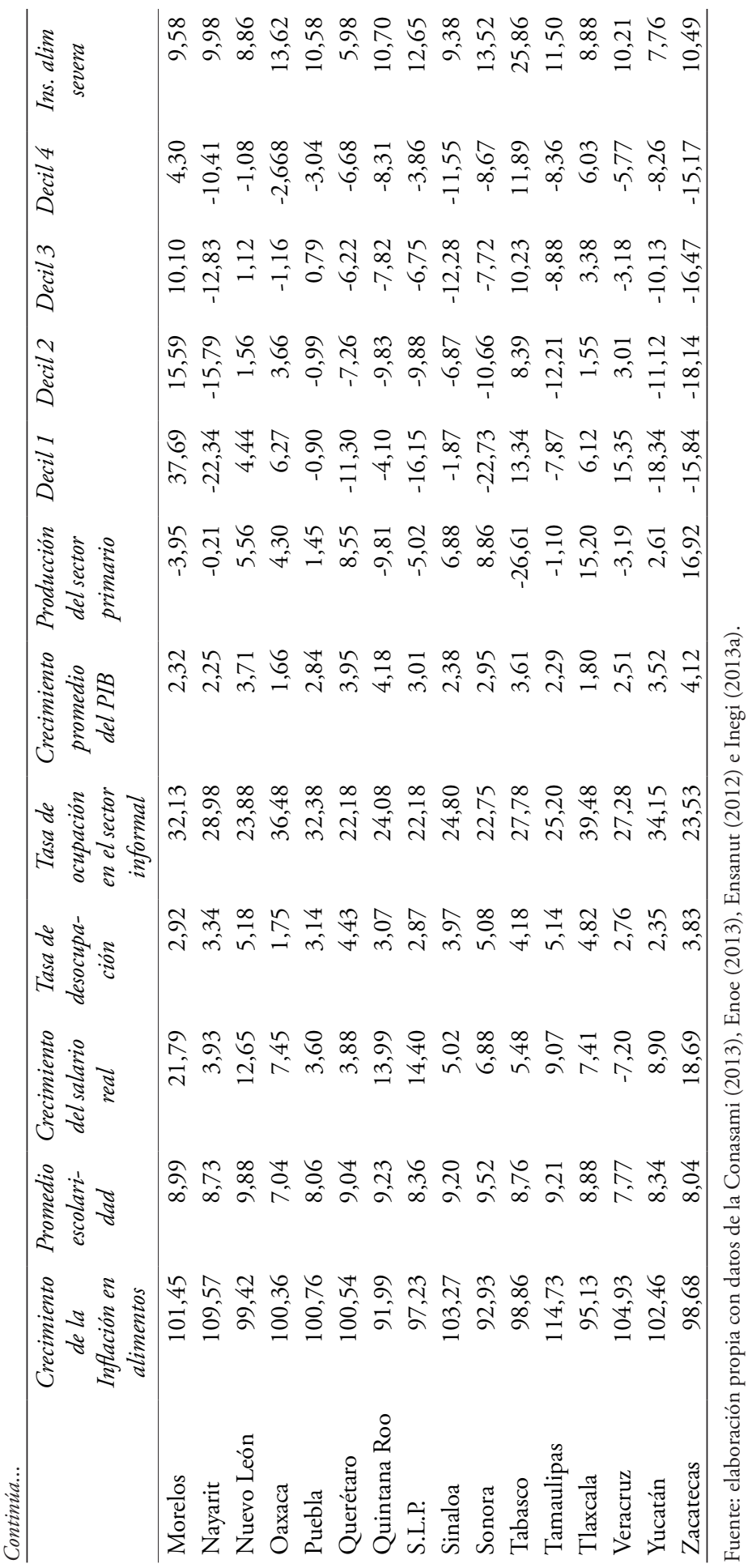


factores explicativos de la inseguridad alimentaria severa en los estados de México.

Por otra parte, respecto a la variable de escolaridad, que representa una aproximación al grado de capacitación de las personas para realizar sus actividades, se encontró en términos medios que dicha variable presenta una relación inversa con la variable IAS, lo cual puede ejemplificarse con entidades como el Distrito Federal y Nuevo León, quienes muestran los niveles de escolaridad más elevados (10.6 y 9.9 años, respectivamente) entre los 32 estados; a su vez, se ubican entre las entidades con índices de IAS por debajo del promedio nacional $(11,7 \%)$, lo cual es consistente con el hecho de que un nivel de instrucción más alto se asocia positivamente con niveles de IAS más bajos; o bien, que una mayor capacitación contribuye con niveles de IAS menores. Se puede observar que estados como Chiapas, Oaxaca, Guerrero y Michoacán, que presentan los niveles de escolaridad más reducidos, por debajo de los ocho años en promedio, muestran niveles de IAS por arriba de la media nacional.

En lo relativo a la variable del salario real, donde se esperaría una relación inversa de éste con el indicador de la IAS, resulta que estados como Veracruz, Distrito Federal, Hidalgo y Baja California Sur experimentaron una caída significativa de sus salarios reales, en términos promedio, durante el periodo 2000-2012, sin embargo, sus niveles de IAS se ubicaron por debajo del promedio nacional $(11,7 \%)$, lo que da una primera evidencia de que el crecimiento del salario real en las entidades federativas de México no contribuye de manera significativa al combate de la IAS como se podría suponer.

Por otro lado, al analizar la variable de la tasa de desocupación general y la de ocupación en el sector informal, se aprecia que éstas no tienen un impacto significativo en el nivel de la IAS, esto es, existen entidades federativas que por una parte presentan tasas de desempleo y de ocupación en el sector informal elevadas, y por otra, muestran niveles de IAS cercanos a la media nacional, tal es el caso de Tlaxcala, Estado de México y Guanajuato, que registraron tasas de ocupación en el sector informal de 39,$5 ; 35,0$ y $32,6 \%$, respectivamente, lo que sugiere que el dinámico proceso de absorción de mano de obra por parte del sector informal de la economía no ha sido determinante en el aumento promedio de la población que se encuentra en situación de IAS de los diferentes estados de México.

Otro indicador relevante en el estudio de la IAS en esta investigación es el del crecimiento de la actividad económica de los estados. Al respecto se tiene que durante el periodo de 2000-2012 las entidades que registraron los mayores crecimientos porcentuales promedio de su producto interno bruto (PIB) fueron los de Baja California Sur $(4,6)$, Aguascalientes 
$(4,3)$, Quintana Roo (4,2), Zacatecas $(4,1)$ y Querétaro $(4,0)$. No obstante, este comportamiento favorable de sus economías no se ve claramente reflejado en los niveles de IAS, los cuales fueron similares al promedio nacional, excepto Querétaro, donde sí se observaría una relación directa entre crecimiento económico y bajo nivel de IAS.

Campeche, Oaxaca, Michoacán, Tlaxcala y el Distrito Federal tuvieron las menores tasas de crecimiento de su PIB, por otra parte, su nivel de IAS no resulta tan alto como se podría esperar; al respecto llama la atención el caso de Tlaxcala con una IAS muy por debajo del promedio nacional y con un crecimiento económico promedio, en el periodo referido, de apenas 1,8 por ciento.

Por otra parte, considerando que el crecimiento de la actividad productiva per cápita del sector primario de las economías estatales influirá de manera positiva en la seguridad alimentaria de sus poblaciones, se observó que los estados de Zacatecas, Tlaxcala, Chihuahua, Sonora y Querétaro presentaron las tasas más altas de crecimiento durante el periodo 2005-2010; en tanto que Tabasco, Baja California Sur, Colima, Quintana Roo y el Distrito Federal mostraron los crecimientos más bajos, por cierto negativos.

En este sentido, el contraste de ambos casos en relación a los niveles de IAS sugieren para la mayoría de los estados una relación inversa entre estas dos variables, es decir, a mayor crecimiento de la actividad productiva primaria per cápita se observaron menores niveles en la IAS y, viceversa, niveles de crecimiento reducidos, incluso negativos, están asociados con mayores índices de IAS.

Ahora, al considerar la evolución del nivel de ingreso de los estados, esto a partir de la variable del ingreso por deciles donde además se espera una relación inversa entre el crecimiento del ingreso y la proporción de familias en condición de IAS, se distingue que para el decil I, ${ }^{7}$ estados como Morelos, Veracruz, Chiapas, Tabasco y Michoacán han mostrado un mayor crecimiento en el mismo durante el periodo 2005-2010; en tanto que Jalisco, Sonora, Colima, Nayarit y Yucatán presentaron crecimientos negativos para dicha variable. Así, en el primer grupo de estados se observaron niveles elevados de IAS y no en el segundo grupo, donde de hecho los estados de Jalisco, Colima, Nayarit y Yucatán se encontraron con niveles de IAS por debajo de la media nacional.

${ }^{7}$ Los deciles I, II y III corresponden a la percepción de ingresos menor a un salario mínimo, los deciles IV, V Y VI equivalen a la obtención de más de un salarios mínimos, mientras que el decil VII y VIII representa el ingreso de más de dos salarios mínimos, el decil IX reporta un ingreso mayor a cuatro salarios mínimos y finalmente el decil X con una percepción mayor a siete salarios mínimos (Inegi, 2011). 
Un caso especial que llama la atención es el del estado de Tamaulipas que aunque por una parte presentó la tasa de inflación en alimentos más elevada entre los estados, durante el periodo de 2000-2012, por otra se ubicó en los primeros lugares en tasas de desocupación promedio para el mismo periodo, presentó un grado de IAS no tan elevado en términos relativos $(11,5 \%)$.

Asimismo, se detectaron casos como el de Chiapas donde sus diversos indicadores económicos son consistentes con lo esperado, esto es, mientras Chiapas fue el estado con el menor nivel de escolaridad (6,8 años), elevada inflación en alimentos (101,8\% durante 2000-2012), crecimiento promedio del PIB (2\% en 2000-2011), y una fuerte reducción de la producción del sector primario per cápita (-4,7\% en 2005-2010), su nivel de IAS durante 2012 resultó de los más elevados (15,4\%), sólo por debajo de Tabasco (25,9\%), Campeche (18,3\%), Guerrero (18\%) y Michoacán $(16,3 \%)$.

\section{Modelo econométrico y resultados}

Es importante destacar que la finalidad de este análisis no es la predicción acerca del comportamiento de las variables en estudio sino únicamente conocer aquellos factores que, en términos medios, contribuyen a una mejor explicación del fenómeno de la inseguridad alimentaria severa en los estados de México.

El modelo de inseguridad alimentaria especificado ${ }^{8}$ y estimado en primera instancia es el siguiente: ${ }^{9}$

$$
\begin{aligned}
& Y=49.06-0.14 X_{1}-2.20 X_{2}-0.02 X_{3}+0.20 X_{4}-0.08 X_{5}-0.82 X_{6}-0.19 X_{7} \\
& +0.13 X_{8}-0.42 X_{9}+0.17 X_{10}+0.21 X_{11} \\
& \begin{array}{llllll}
(0.0059) & (0.1705) & (0.093) & (0.8255) & (0.8239) & (0.5224)
\end{array} \\
& \begin{array}{lll}
(0.2245) & (0.0212) & (0.2970)
\end{array} \\
& \begin{array}{lll}
(0.2908) & (0.7122) & (0.5352)
\end{array}
\end{aligned}
$$

Donde las cifras entre paréntesis corresponden a los valores de probabilidad, o $p$-values, ${ }^{10}$ los cuales permiten evaluar la significancia de los

${ }^{8}$ Este modelo considera información de corte transversal y series de tiempo (sección cruzada).

${ }^{9}$ En la estimación del modelo se hizo uso del software estadístico y econométrico: EViews y $\mathrm{R}$-project. $\mathrm{R}$ es un lenguaje y entorno para computación y gráficos estadísticos, proporciona una amplia variedad de procedimientos estadísticos (modelos lineales y no lineales, pruebas estadísticas clásicas, análisis de series temporales, clasificación, agrupamiento...), así como técnicas gráficas. Veáse: http://www.r-project.org/

${ }^{10}$ El p-value es una estimación de la probabilidad del error tipo I en una prueba de hipótesis donde se considera la probabilidad de rechazar la hipótesis nula cuando ésta es cierta y el valor crítico de dicha prueba se determina a partir de los valores de la muestra. 
parámetros estimados del modelo. Además, es importante recordar que el análisis de la estimación del modelo se realiza en términos promedio para los 32 estados de la república mexicana.

Por otra parte, para determinar si el modelo tiene una correcta especificación se recurrió a la prueba Reset de Ramsey, misma que arrojó un valor de probabilidad del estadístico $\mathrm{F}$ de 0.0081 , el cual es menor a 0.05 , y por tanto, sugiere que la hipótesis nula de correcta especificación debe ser rechazada considerando un nivel de significancia de 5 por ciento. Adicionalmente, la presencia de una gran cantidad de coeficientes no significativos (lo que se observa a partir de los p-values mayores a 0.05 en el modelo estimado) sugirió la incorporación de una especificación distinta del modelo. Es así que se consideró el planteamiento de un modelo $\log \operatorname{lin}^{11}$, el cual implica que la relación en términos de las variables originales es la forma funcional: ${ }^{12}$

$$
Y=e^{\beta_{\mathrm{e}}+\beta_{1} X_{1}+\beta_{2} X_{2}+\varepsilon}
$$

Donde $\beta_{\mathbf{0}}, \beta_{\mathbf{1}} y \beta_{\mathbf{2}}$ son los parámetros a estimar, $\mathrm{X}_{1} \mathrm{y} \mathrm{X}_{2}$ las variables explicativas del modelo, $\mathrm{Y}$, la variable inseguridad alimentaria severa (IAS) de los estados de México y $\varepsilon$ es el término de error aleatorio del modelo econométrico. A su vez, la estimación de este modelo por el método de mínimos cuadrados ordinarios (MCO) fue la siguiente.

$$
\begin{gathered}
\ln Y=3.75-0.15 X_{\mathbf{z}}-0.01 X_{7} \\
(0.0000)(0.0066) \quad(0.0088)
\end{gathered}
$$

Con base en esta especificación y estimación se encontró que la escolaridad $\left(\mathrm{X}_{2}\right)$ y la producción del sector primario per cápita $\left(\mathrm{X}_{7}\right)$ son los principales factores explicativos de la IAS para los 32 estados de México, los cuáles en conjunto determinarían alrededor de un $33 \%\left(\mathrm{R}^{2}=0.33\right)^{13}$ de dicha problemática, además de que ambas variables presentan signo

\footnotetext{
${ }^{11}$ Un modelo log-lin plantea que la relación entre la variable respuesta $Y$ y las variables explicativas $\left(\mathrm{X}^{\prime} \mathrm{s}\right)$ responde a una función de tipo exponencial.

${ }^{12}$ Cabe resaltar que bajo ésta suposición funcional es posible la validación de los distintos supuestos que condicionan el diagnóstico del modelo econométrico ante el empleo del método de MCO.

${ }^{13}$ Además, los resultados correspondientes a las pruebas de heteroscedasticidad, autocorrelación y normalidad de los errores indican que no existen inconsistencias al respecto. La prueba de Breusch -Pagan-Godfrey y White mostraron un p-value de 0.92 y 0.96 , respectivamente, lo que sugiere homogeneidad de varianza en los errores. Adicionalmente, la prueba de Autocorrelación de BreuschGodfrey (LM), mostró un valor p de 0.30 con lo que no hay evidencia de autocorrelación serial de los errores. Por otra parte, las pruebas de normalidad de jarque-Bera y Shapiro Wilk presentaron p-values de 0.30 y 0.25 con lo que se satisface el supuesto de normalidad.
} 
negativo, es decir, que tendrían una relación inversa con la variable de la IAS, lo que es consistente con el planteamiento teórico correspondiente.

En este sentido, variables como el nivel de ingreso estatal, la inflación en alimentos, el crecimiento del producto interno bruto (PIB), la tasa de desempleo y del empleo en el sector informal de las economías estatales, en términos promedio, no constituyen factores determinantes en la explicación del problema de la IAS a nivel estatal para México de acuerdo con la estimación del modelo econométrico propuesto en esta investigación.

De esta manera, vale la pena remarcar que el panorama que se presenta en variables como escolaridad media y producción del sector primario per cápita en los estados mexicanos es poco alentador. En México, el grado promedio de escolaridad de la población es de 8,7 años, lo que es equivalente, en el mejor de los casos, a estudios de secundaria. $\mathrm{Al}$ respecto, en entidades como el Estado de México, Baja California, Sonora, Coahuila y Baja California Sur, que presentan grados de escolaridad que van de los 9,2 a los 9,6 años, cifras equivalentes a poco más de la secundaria concluida, no han podido resolver de forma adecuada el problema de la IAS que alcanza porcentajes entre el 10,7 y 13,5\% de los hogares en dichas entidades.

Por su parte, estados como Chiapas, Guanajuato, Guerrero, Michoacán y Veracruz que tienen promedios de escolaridad de entre 6,8 y 7,9 años presentan niveles de IAS que alcanzan porcentajes entre 10,21 y $17,97 \%$ de sus poblaciones (ver cuadro 1 ).

A su vez, en relación a la producción per cápita del sector primario de las economías estatales se puede observar que el crecimiento de esta variable durante el periodo 2005-2010 recurrentemente ha presentado cifras negativas, con excepciones como Zacatecas $(16,9 \%)$, Tlaxcala $(15,2 \%)$, Chihuahua (12,7\%), Sonora $(8,9 \%)$ y Querétaro $(8,6 \%)$, lo que puede explicar en buena medida la creciente dependencia alimentaria que ha mostrado el país mexicano, principalmente respecto a Estados Unidos de Norteamérica; esta situación sin duda está asociada a la fuerte contracción del sector agropecuario mexicano en las últimas décadas.

Al respecto, Antón (2013) y González y Orrantia (2006) han establecido que la problemática del campo mexicano, más que ser resultado de la política subsidiaria hacia ese sector o de la mayor integración al comercio internacional, se debe principalmente a la insuficiente inversión pública y privada en servicios de apoyo que promuevan el avance técnico y capacitación, la ampliación de la infraestructura de riego y transporte, la organización para la compra de insumos y para la venta de la producción, seguro agrícola y crédito, así como la intensificación del proceso productivo y la productividad del sector. 


\section{Conclusiones}

La revisión de las teorías de Sen $(1981,1983)$ sugiere que para poder explicar el fenómeno de la inseguridad alimentaria es necesario considerar variables como el nivel de ingreso y su distribución, la tasa de empleo, las capacidades y titularidades o derechos de los individuos, ya que éstas se encuentran estrechamente vinculadas a la problemática de estudio.

Es así que en esta investigación se consideró adecuado tomar en cuenta las variables de inflación doméstica en los alimentos, salario real, escolaridad, tasa de desocupación general, tasa de ocupación en el sector informal, crecimiento del producto interno bruto, crecimiento de la producción per cápita en el sector primario y el ingreso poblacional por deciles con la finalidad de explicar aquellos factores que pudieran tener mayor incidencia en la problemática de la inseguridad alimentaria de los estados de México. No obstante, el modelo econométrico estimado mostró que las variables que más contribuyeron en la explicación del problema de la inseguridad alimentaria severa en los estados de México durante 2012 fueron la escolaridad media y crecimiento del sector primario per cápita de las economías estatales.

La variable escolaridad, que resultó significativa en el modelo estimado, presenta una estrecha vinculación con el enfoque de capabilities en el sentido de que un nivel adecuado de escolaridad permitiría (u obstaculizaría, en caso contrario) a los individuos para tener mayores posibilidades de realizar o alcanzar las categorías relativas a las principales capabilities propuestas por Nussbaum (2000 y 2003) que a su vez permitirían un mayor nivel de bienestar a las personas. En este sentido, la toma de decisiones informada por parte de los individuos sin duda contribuiría en la mejoría de su calidad de vida. Al respecto, una decisión que es fundamental es la relativa a la elección de una alimentación suficiente y de calidad. En este sentido, la Ensanut (2012) plantea que en el corto plazo es fundamental la orientación que en nutrición familiar se pueda llevar a la población, tomando en cuenta las necesidades de los individuos con bajos niveles de alfabetismo y presupuestos limitados.

En este contexto, el Banco Mundial (2005) sugirió que el combate a los problemas de pobreza e inseguridad alimentaria debe considerar de manera prioritaria $i$ ) el acceso universal a la educación, ii) la ampliación de las redes de protección social, iii) la promoción de la equidad de género, iv) la creación de empleo, $v$ ) el aumento de los programas de nutrición, y vi) la promoción de la inversión en el sector agrícola. Lo que pone de manifiesto la trascendencia del factor educativo como un mecanismo fundamental en el control del fenómeno de la inseguridad alimentaria. 
Drèze y Sen (1989: 22-25) al analizar el caso de Kerala, uno de los estados más pobres de la India, establecen que éste ha alcanzado una expectativa de vida extraordinariamente elevada, incluso por arriba de los estados más ricos como Punjab y Haryana. Adicionalmente se argumenta que:

el alto nivel de alfabetización de Kerala ha sido acompañado de una participación social activa, traducida en demandas políticas y sociales bien articuladas, que a la postre ha derivado en la introducción de programas innovadores de salud y alimentación de mayor cobertura y calidad (siendo el único estado donde la distribución pública de alimentos trasciende las zonas urbanas para apoyar significativamente poblaciones rurales), así como en una serie de cambios institucionales que han favorecido en particular la legislación sobre salarios y reformas agrarias.

Por otra parte, la variable producción del sector primario también resultó significativa en términos estadísticos, lo que sugiere la relevancia que puede tener el crecimiento sostenido de la producción (en términos per cápita) del sector primario en el control de la inseguridad alimentaria severa de las economías estatales, puesto que es en este sector donde se origina gran parte la producción de alimentos del país. En este sentido, sería conveniente impulsar y enfocar la inversión, tanto pública como privada, de este sector, fundamentalmente en la capacitación de los productores con la finalidad de incentivar el cambio técnico, la ampliación de la infraestructura de riego y transporte, el desarrollo de programas de agricultura familiar donde pequeños agricultores reciban incentivos fiscales y agropecuarios para producir frutas y verduras con el objetivo de distribuirlas en poblaciones vulnerables, así como ampliar el crédito y aseguramiento de las actividades agropecuarias bajo reglas específicas de beneficios y obligaciones por parte de los productores y autoridades involucradas. Dichas medidas contribuirían a la intensificación del proceso productivo y la productividad del sector siguiendo el planteamiento de González y Orrantia (2006) y la Instituto Nacional de Salud Pública (2012).

\section{Fuentes consultadas}

Alarcón, Gloria y Cristina Guirao (2013), "El enfoque de las capacidades y las competencias transversales en el EEES", Historia y comunicación social, vol. 18, Universidad Complutense de Madrid, Madrid, pp. 145-157.

Antón, Jesús (2013), "Sector agrícola y desarrollo rural”, Getting it right: Una agenda estratégica para las reforma en México, OCDE publishing, 
<http://www.keepeek.com/Digital-Asset-Management/oecd/ governance/getting-it-right/sector-agricola-y-desarrollorural_9789264190375-15-es\#page1>, 10 de enero de 2015.

Banco Mundial (2005), "Lograr la educación primaria universal para 2015”, Banco Mundial, Washington, <http:/www.bancomundial.org/odm/educacion.html >, 8 de julio de 2015 .

Cabrera, Juan Carlos (2009), "Inflación, salarios y nivel de vida”, Economía Informa, núm. 357, Universidad Nacional Autónoma de México, México, pp. 62-70.

Cabrera, Juan Carlos, Abelardo Aníbal Gutiérrez y Rubén Antonio (2005), Introducción a los indicadores económicos y sociales de México, Universidad Nacional Autónoma de México, México.

Camberos, Mario (2000), “La seguridad alimentaria de México en el año 2030", Ciencia Ergo Sum, 7 (1), Universidad Autónoma del Estado de México, Toluca, pp. 49 -55.

Coordinación de Seguridad Alimentaria y Acceso a Oportunidades Económicas (2010), "Mapa de vulnerabilidad a la inseguridad alimentaria", Coordinación de Seguridad Alimentaria y Acceso a Oportunidades Económicas, Lima, Perú. <http://americalatina. landcoalition.org/sites/default/files/Mapa\%20de\%20Vulnerabilidad\%20a\%20la\%20Inseguridad\%20Alimentaria.pdf>, 20 de agosto de 2015.

Conasami (Comisión Nacional de los Salarios Mínimos) (2013), "Salarios mínimos", Comisión Nacional de Salarios Mínimos, México, $<$ www.conasami.gob.mx/salarios_minimos.html>, 28 de junio de 2013.

Coneval (Consejo Nacional de Evaluación de la Política de Desarrollo Social) (2015a), Resultados de pobreza en México 2012 a nivel nacional y por entidades federativas, Consejo Nacional de Evaluación de la Política de Desarrollo Social, México, <http://www. coneval.gob.mx/Medicion/Paginas/Medici\%C3\%B3n/Pobreza\%202012/Pobreza-2012.aspx >, 7 de enero de 2015.

Coneval (Consejo Nacional de Evaluación de la Política de Desarrollo Social) (2015b), Lineamientos y criterios generales para la defi- 
nición, identificación y medición de la pobreza, Diario Oficial de la Federación, 16 de junio de 2010, Consejo Nacional de Evaluación de la Política de Desarrollo Social, México, <http://www. coneval.gob.mx/rw/resource/coneval/med_pobreza/DiarioOficial/DOF_lineamientos_pobrezaCONEVAL_16062010.pdf>, 7 de enero de 2015.

Coneval (Consejo Nacional de Evaluación de la Política de Desarrollo Social) (2010), Dimensiones de la seguridad alimentaria: Evaluación Estratégica de Nutrición y Abasto, Consejo Nacional de Evaluación de la Política de Desarrollo Social, México.

Cuéllar, José Alberto (2011), Programa de Seguridad Alimentaria: experiencias en México y otros países, Cepal, México.

Drèze, Jean y Amartia Sen (1989), Hunger and public action, Oxford University Press, Oxford.

Enoe (Encuesta Nacional de Ocupación y Empleo) (2013), "Encuesta en Hogares", Inegi, México, <www3.inegi.org.mx/sistemas/Infoenoe/Default_CONAPO.aspx?s=est\&c=26227\&p=>, $26 \mathrm{de}$ abril de 2013.

Ensanut (Encuesta Nacional de Salud y Nutrición) (2012), "Encuesta Nacional de Salud y Nutrición. Resultados nacionales", primera edición, Instituto Nacional de Salud Pública, México, <ensanut. insp.mx/informes/ENSANUT2012ResultadosNacionales.pdf>, 7 de julio de 2013.

FAO (Organización de las Naciones Unidas para la Agricultura y la Alimentación) (2011), "Una introducción a los conceptos básicos de la seguridad alimentaria: información para la toma de decisiones", FAO, México, <www.fao.org/docrep/014/al936s/al936s00. pdf>, 25 de junio de 2013.

Ginja, Victoria (2008), Diagnóstico, modelo y atlas municipal de seguridad alimentaria en Bolivia, Programa Mundial de Alimentos, Bolivia.

González, Adrián y Manuel Alejandro Orrantia (2006), "Los subsidios agrícolas de México”, Agricultura Técnica en México, 32 (3), Instituto Nacional de Investigaciones Forestales, Agrícolas y Pecuarias, México, pp. 323-331. 
Hurtado, Jesús (2011), "Análisis de los cambios en las políticas de seguridad alimentaria y su impacto en el sistema agroalimentario venezolano entre los periodos 1994-1998 y 1999-2007”, tesis de doctorado, Universidad Central de Venezuela, Venezuela.

Inegi (Instituto Nacional de Estadística y Geografía) (2015), "Precios e inflación: inflación acumulada anual", índice general, Inegi, México, <http://www.inegi.org.mx/sistemas/bie/>, 7 de enero de 2015 .

Inegi (Instituto Nacional de Estadística y Geografía) (2013a), "Banco de Información Económica”, Inegi, México, <http://www.inegi.org. $\mathrm{mx} /$ sistemas/bie/?idserPadre $=11100070001000300080 \# \mathrm{D} 111$ $00070001000300080>, 24$ de julio de 2013.

Inegi (Instituto Nacional de Estadística y Geografía) (2013b), "Encuesta Nacional de Ocupación y Empleo”, Inegi, México, <http://www3. inegi.org.mx/sistemas/Infoenoe/ Default_CONAPO.aspx?s= est\&c=26227\&p=>, 24 de julio de 2013.

Inegi (Instituto Nacional de Estadística y Geografía) (2013c), "Cuentas nacionales", Inegi, México, <http://www.inegi.org.mx/sistemas/ bie/?idserPadre=10200026>, 26 de julio de 2013.

Inegi (Instituto Nacional de Estadística y Geografía) (2013d), "Encuesta Nacional de Ingresos y Gastos de los Hogares (ENIGH) 2012", Boletín de prensa núm. 278/13, Inegi, Aguascalientes, p. 1/2, <http://www.inegi.org.mx/inegi/contenidos/espanol/prensa/ boletines/boletin/Comunicados/Especiales/2013/Julio/comunica5.pdf>, 25 de junio de 2013.

Inegi (Instituto Nacional de Estadística y Geografía) (2012), Anuario de estadistica por entidad federativa 2012, Inegi, México.

Inegi (Instituto Nacional de Estadística y Geografía) (2011), "Contenidos", Inegi, México, <http://www.inegi.org.mx/inegi/contenidos/.../2011/Julio/comunica2.pdf>, 13 de junio de 2013.

Instituto Nacional de Salud Pública (2013), "Encuesta Nacional de Salud y Nutrición (Ensanut), Seguridad alimentaria”, Instituto Nacional de Salud Pública, México, <ensanut.insp.mx/informes/ENSANUT2012ResultadosNacionales.pdf>, 25 de junio de 2013. 
Instituto Nacional de Salud Pública (2012), "Encuesta Nacional de Salud y Nutrición. Resultados nacionales", Instituto Nacional de Salud Pública, México, <ensanut.insp.mx/informes/ENSANUT2012ResultadosNacionales.pdf $>, 7$ de julio de 2013.

Malthus, Thomas Robert (1798), "An Essay on the Principle of Population, as it affects the Future Improvement of Society: with remarks on the Speculations of Mr. Godwin, M. Condorset, and other writers", J. Johnson, London, <http://www.esp.org/books/malthus/ population/malthus.pdf>, 26 de agosto de 2015 .

Melgar, Hugo, Ana Claudia Zubieta, Enriqueta Valdez, Bárbara Whitelaw y Lucia Kaiser (2005), "Validación de un instrumento para vigilar la inseguridad alimentaria en la Sierra de Manatlán, Jalisco", Salud Pública de México, 47 (6), Instituto Nacional de Salud Pública, Cuernavaca, pp. 413-422.

Nebel, Mathias, Pedro Flores y Ma. Teresa Herrera (2014), Desarrollo como libertad en América Latina, fundamentos y aplicaciones, Universidad Iberoamericana, México.

Nikos, Alexandratos y Jelle Bruinsma (2012), "World agriculture towards 2030/2050: the 2012 revision", ESA Working Paper 12-03, Organización de las Naciones Unidas para la Agricultura y la Alimentación (FAO), México, <http://www.fao.org/docrep/016/ ap106e/ap106e.pdf>, 12 de enero de 2015.

Nussbaum, Martha (2000), Women and human development. The capabilities approach, Cambridge University Press, Cambridge.

Nussbaum, Martha (2003), "Capabilities as fundamental entitlements: Sen and social justice", Feminist Economics, 9 (2-3), Routledge, Houston, pp. 33-59.

OSAN (Observatorio de Seguridad Alimentaria y Nutricional) (2011), Observatorio de Seguridad Alimentaria y Nutricional de Colombia: Componentes y lineamientos para su implementación, Instituto Nacional de Salud, Medellín.

Robeyns, Ingrid (2005), "The capability approach: a theoretical survey”, Journal of Human Development, 6 (1), Routledge, Inglaterra, 
pp. 93-117, <http://www.tandfonline.com/doi/pdf/10.1080/14 6498805200034266>, 3 de agosto de 2015.

Secretaría de Salud (2012), Encuesta Nacional de Salud y Nutrición 2012. Resultados Nacionales, Secretaría de Salud, México, <http://ensanut.insp.mx/informes/ENSANUT2012ResultadosNacionales. pdf>, 7 de julio de 2013 .

Sen, Amartya (1983), "Development: which way now?", The Economic Journal, vol. 93, Jstor, pp. 745-762.

Sen, Amartya (1981), "Ingredients of famine analysis: availability and entitlement", Quarterly Journal of Economics, 96 (3), Jstor, pp. 433-464.

Sulo, Timoteo y Sharon Chelangat (2012), "An econometrics assessment of food security estimation using fuzzy logics: acase in the arid and semiarid lands of Kenya”, Global Journal of Science Frontier Research, 12 (9), Open Association of Research Society, pp. 1-12.

Torres, Felipe (2003), "Seguridad Alimentaria: Seguridad Nacional", Universidad Nacional Autónoma de México, México, <http:// ru.iiec.unam.mx/1762/1/SegAlimentaria.pdf >, 6 de julio de 2015.

Torres, Felipe (2002), "Aspectos regionales de la seguridad alimentaria en México", Revista de Información y Análisis, núm. 22, Inegi, México, pp. 15-26.

Zegarra, Eduardo y Jorge Tuesta (2009), "Shock de precios y vulnerabilidad alimentaria de los hogares peruanos", Documento de trabajo 55, Grupo de Análisis para el Desarrollo, Lima, Perú, <http:// ageconsearch.umn.edu/bitstream/59688/2/ddt55.pdf>, 7 de julio de 2013.

Recibido: 5 de marzo de 2014. Corregido: 5 de diciembre de 2014. Aceptado: 3 de septiembre de 2015.

Miguel Ángel Díaz-Carreño. Doctor en ciencias con especialidad en economía por el Colegio de Posgraduados, México. Actualmente es profesor-investigador de la Facultad de Economía de la Universidad Au- 
tónoma del Estado de México. Es miembro del Sistema Nacional de Investigadores, nivel I. Sus líneas de investigación son estadística aplicada, macroeconomía aplicada y economía agrícola. Entre sus últimas publicaciones destacan: "Frontera tecnológica y productividad total de los factores de las regiones de México", Región y Sociedad, núm. 57, El Colegio de Sonora, Hermosillo, pp. 5-26 (2013); "De la recesión a la recuperación: Producción y empleo en México y el Estado de México", Problemas del Desarrollo, núm. 173, Universidad Nacional Autónoma de México, México, pp. 133-162 (2013); "Análisis de las depreciaciones extremas del tipo de cambio de México a partir del Proceso Poisson no Homogéneo", Ciencia Ergo Sum, XVIII (3), Universidad Autónoma del Estado de México, Toluca, pp. 243-248 (2012); "Interacciones económico-financieras Brasil-México: ¿̇cuál es su grado de integración?”, Perfiles Latinoamericanos, núm. 39, Facultad Latinoamericana de Ciencias Sociales, México, pp. $117-150$ (2012).

Alejandra Díaz-Bustamante. Licenciada en Relaciones Económicas Internacionales por la Facultad de Economía de la Universidad Autónoma del Estado de México.

Mayte Sánchez-León. Licenciada en Relaciones Económicas Internacionales por la Facultad de Economía de la Universidad Autónoma del Estado de México. 